\title{
Prozessmanagement im Krankenhaus: Spielarten und deren Wirkungsweisen
}

\section{Jürgen Merz, Silke Bucher, Johannes Rüegg-Stürm}

Bisher in dieser Reihe in loser Folge erschienene Artikel in SÄZ 23, 27-28 und 45 (alle 2007) sowie 7, 14, 23 und 33/2008 auf www.saez.ch.

Forschungsprogramm HealthCare Excellence

Silke Bucher, David Kurz, Jürgen Merz, Prof. Dr. Johannes Rüegg-Stürm,

Dr. Harald Tuckermann,

Dr. med. Jürg Unger-Köppel,

Dr. Widar von Arx

Institut für Betriebswirtschaft, St. Gallen
Korrespondenz:

Prof. Dr. oec. Johannes Rüegg-Stürm Institut für Betriebswirtschaft

Dufourstrasse 40a

CH-9000 St. Gallen

Tel. 0712242323

Fax 0712242355

johannes.rueegg@unisg.ch

www.healthcaremanagement.ch

www.ifb.unisg.ch
Mit Versuchen, die Ausgaben im Gesundheitswesen zu beschränken, ist das Krankenhaus als einer der zentralen Erbringer von Gesundheitsleistungen seit längerem in den Mittelpunkt von Effizienz- und Effektivitätsbestrebungen gerückt. Nicht selten wird dabei der Ruf nach «Prozessmanagement» laut, verbunden mit dem Wunsch, die im System vorhandenen Kostensenkungsund Leistungspotentiale über dieses Konzept auszuschöpfen. In der Praxis zeigt sich dann, dass «Prozessmanagement» in Krankenhäusern in einer Vielzahl von Varianten verfolgt wird. Jede dieser Varianten kann ihre Berechtigung haben. Um jedoch Verwirrung unter den Mitarbeitenden oder ein Verpuffen wertvoller Anstrengungen zu vermeiden, ist es wichtig, sich über die jeweils angemessenen Varianten und deren Wirkungsweisen zu verständigen.

\section{Prozessmanagement ist nicht gleich Prozessmanagement}

Ein Grossteil der Krankenhäuser ist heute bereits mit Prozessmanagement in Berührung gekommen, sei es in Form eher konzeptioneller Debatten oder in konkreten Umsetzungsversuchen. Dabei zeigt sich jedoch: Die Verständnisse darüber, was Prozessmanagement ausmacht, gehen weit auseinander. Und nicht immer sind sich die Beteiligten über die unterschiedlichen Sichtwesen im Klaren. Dies wiederum führt leicht zu Kommunikationsproblemen und Missverständnissen im Vorfeld von Prozessmanagementinitiativen und kann solchen Vorhaben von Beginn an viel Energie nehmen. Oder aber, die Beteiligten können aufgrund der Vielheit an Verständnissen nicht abschätzen, welche der Varianten zur Erreichung der gesetzten Ziele, bspw. der so häufig geforderten Kosten- und Effektivitätseffekte, beitragen könnte.

Ziel dieses Artikels ist es daher, zunächst die geläufigsten Prozessmanagementverständnisse der Praxis zu beschreiben und zu systematisieren. Diesen Verständnissen wird schliesslich unser Ansatz der «Evolutionären Prozessoptimierung» gegenübergestellt, eine Spielart, die sich insbesondere in komplexen Organisationen wie dem Krankenhaus für die Bewältigung der anstehen-

\section{Gestion des processus}

\section{à I'hôpital: aspects et modes}

\section{de fonctionnement}

Les tentatives visant à limiter les coûts de la santé placent depuis longtemps I'hôpital, en tant que I'un des fournisseurs principaux de prestations, au centre des efforts pour augmenter l'efficacité économique des soins. Dans ce contexte, il n'est pas rare d'entendre parler de «gestion des processus» et, partant, du vœu d'épuiser les possibilités du système pour réduire les coûts et optimiser les prestations. Il apparaît toutefois que les hôpitaux abordent ce thème sous des formes multiples et souvent de manière propre à susciter le trouble parmi les collaborateurs. De ce fait, il est important de s'assurer de l'objectif concret visé par la gestion des processus.

den Herausforderungen bewährt hat. Die «Versprachlichung» und Systematisierung der unterschiedlichen Prozessmanagementverständnisse kann dazu beitragen, entsprechende Initiativen von Beginn an kommunizier- und verhandelbar zu machen und zumindest den Mangel an einem gemeinsamen Verständnis als mögliche Hürde für erfolgreiche Vorhaben zu schmälern. Darüber hinaus wird mit der Beschreibung der «Evolutionären Prozessoptimierung» der Boden bereitet für einen Folgeartikel unter gleichnamigem Titel. Dieser wird aufzeigen, auf welche Weise konkret die im Mittelpunkt stehenden Effizienz- und Effektivitätsbestrebungen mit Hilfe einer «Evolutionären Prozessoptimierung» bewältigt werden können.

\section{Spielarten des Prozessmanagements}

Gegenstand des Prozessmanagements sind zwar immer die Prozesse, also die Abläufe, einer Organisation. Dennoch verbindet die Krankenhaus- 
praxis ganz unterschiedliche Vorstellungen und Herangehensweisen mit dem Thema Prozessmanagement. So werden Abläufe einmal relativ abstrahiert, das heisst vom unmittelbaren Alltagsgeschehen weitgehend losgelöst betrachtet, ein anderes Mal wiederum sehr konkret unter die Lupe genommen. Im letzteren Fall fokussiert das Prozessmanagement die alläglichen routinisierten Praktiken und Interaktionsmuster, wie sie sowohl im Austausch und in der Beziehung zwischen den Mitgliedern einer Organisation als auch in Interaktion zwischen den Mitgliedern und ihren Aufgaben stattfinden. Neben den unterschiedlichen Abstraktionsstufen in der Prozessbetrachtung lassen sich die unterschiedlichen Spielarten des Prozessmanagements nach ihrem Wirkungsbereich und nach ihrer Radikalität unterscheiden und in die vier Kategorien Dokumentation, Design, Evolutionäre Optimierung und Revolutionäre Erneuerung gliedern (Abb. 1).

Die Dimension Radikalität beschreibt das Ausmass und die Tiefe der Veränderungen. Sie reicht vom Beibehalten des Status quo in Form einer Dokumentation des Bestehenden bis hin $\mathrm{zu}$ grundlegenden Veränderungen in Form einer Revolutionären Erneuerung. Mit Wirkungsbereich wird die Reichweite der Veränderung angegeben. Diese kann auf einzelne Fachbereiche begrenzt sein, sich aber auch auf ganze Versorgungsregionen erstrecken. Die oben bereits erwähnten vier Spielarten des Prozessmanagements werden im

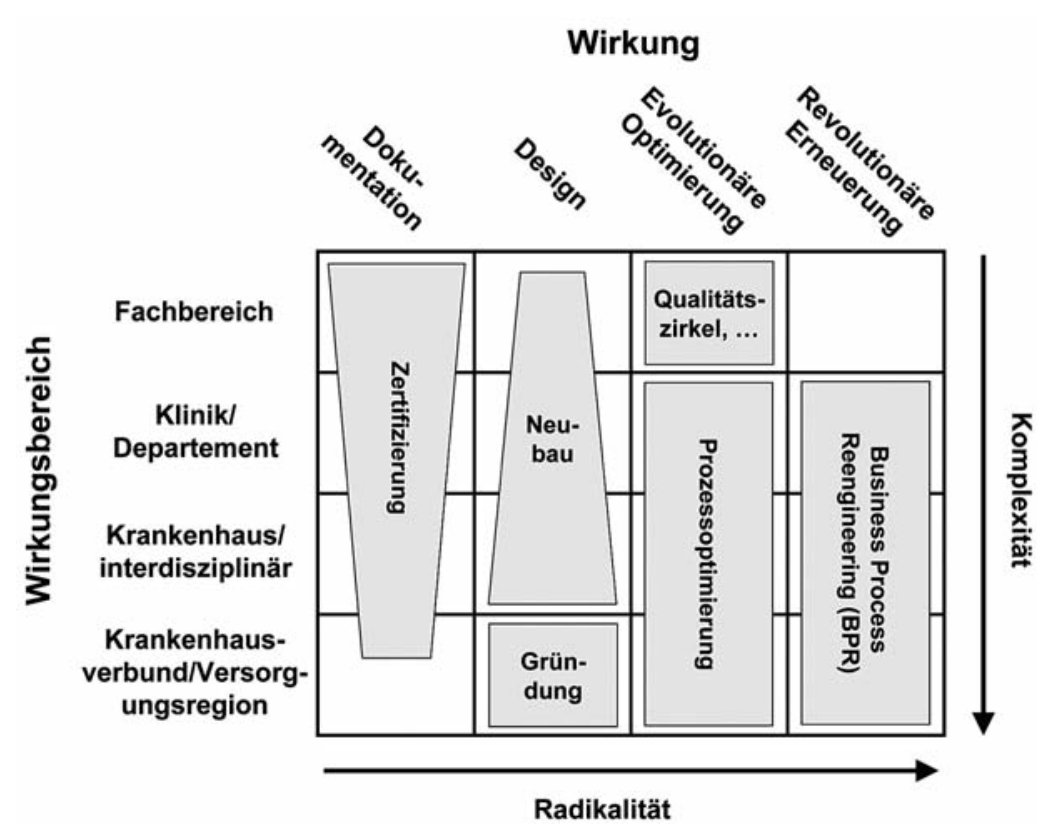

Abbildung 1

Spielarten des Prozessmanagements.
Folgenden kurz skizziert, wobei auf die «Evolutionäre Optimierung» im Anschluss noch ausführlicher eingegangen wird.

\section{Dokumentation}

Im Rahmen von oftmals gesetzlich vorgeschriebenen Qualitäts- und Zertifizierungsmassnahmen (DIN-ISO, EFQM, sanaCERT) wird Prozessmanagement in dieser Spielart im Wesentlichen als Dokumentation von Soll-Prozessen verstanden, die sich in relativ abstrahierter Form an den IstProzessen orientiert. Ziel ist es, Transparenz, rechtliche Sicherheit und Verbindlichkeit über relevante Abläufe zu erhalten und ein angemessenes Qualitätsniveau sicherzustellen. Der Wirkungsbereich erstreckt sich meist auf Prozesse innerhalb von Fachbereichen und Abteilungen. Ein solches Dokumentationsverfahren entfaltet in der Regel nur beschränkte Wirkung auf eine Organisation, weil die Veränderung alltäglicher, routinisierter Interaktionspraktiken dabei nicht im Zentrum der Aufmerksamkeit steht.

\section{Design}

Der Ausgangspunkt dieser Spielart ist die Gründung eines neuen Spitals, einer neuen Klinik oder eines Versorgungsnetzwerks. Die Organisationsarbeit beginnt auf der grünen Wiese, d. h. in einem «geschichtsfreien» Raum. Im Gegensatz zur Spielart «Dokumentation» handelt es sich also bei der Spielart «Design» um eine komplette Neugestaltung von Prozessen, ohne dass bereits Ist-Prozesse vorhanden wären. Idealerweise werden in einem solchen Verfahren zusammen mit den zukünftigen Mitarbeitenden optimale Soll-Prozesse erarbeitet, vergemeinschaftet und dokumentiert. Dies bildet die Grundlage für die Einarbeitung der Mitarbeitenden und die Routinisierung der zukünftigen Abläufe.

\section{Evolutionäre Optimierung}

Unter «evolutionärer Optimierung» verstehen wir ein Vorgehen, das von der gegenwärtigen Organisationspraxis ausgeht und versucht, diese kontinuierlich und sukzessiv weiterzuentwickeln und zu verbessern. Dies wird bspw. im Rahmen sogenannter Qualitätszirkel geleistet. Während diese in der Regel auf Optimierungen innerhalb von Fachbereichen zielen, erstreckt sich die Wirkung einer «evolutionären Prozessoptimierung» über die Grenzen von Fachbereichen hinweg.

\section{Revolutionäre Erneuerung}

Am radikalsten gestaltet sich eine «revolutionäre Erneuerung» von Abläufen. Unter der Bezeichnung Business Process Reengineering oder Business Process Redesign (BPR) hat sie sowohl in der Krankenhausliteratur als auch in der Kranken- 
hauspraxis zwischenzeitlich einen hohen Popularitätswert erreicht. BPR zielt auf die bereichsübergreifende, radikale Umgestaltung von Prozessen innerhalb einer Organisation bzw. über ihre Grenzen hinweg und verspricht dadurch enorme Kostensenkungen und Qualitätssteigerungen. Mit der Fokussierung auf lineare und planbare Prozesse fand BPR vor allem in der Industrie (u.a. Automobil- und Finanzbereich) Anwendung - durchaus erfolgreich. Pauschale Übertragungen des Konzepts auf den Krankenhauskontext sind jedoch meist gescheitert. Gründe dafür gibt es zahlreiche. So sind Patientenprozesse als Gesamtheit aller Aktivitäten der Anamnese, Diagnose und Therapie meist viel weniger linear und weniger planbar als Produktionsprozesse in der Industrie (OP-Prozesse mögen hier eine Ausnahme sein).

Vergleicht man diese hier knapp skizzierten Spielarten des Prozessmanagements unter dem Aspekt einer nachhaltigen Optimierungswirkung oder gar organisationalen Innovation, so fällt auf, dass die Varianten Dokumentation und revolutionäre Erneuerung implizit ein ziemlich simplifizierendes Organisationsverständnis mitführen. Sie reduzieren Abläufe auf eine technisch-sachlogische Tätigkeitsfolge; soziale Beziehungsgefüge sowie Interaktionen zwischen den Mitarbeitenden untereinander und mit ihren Aufgabenbereichen finden wenig Beachtung. Ebenso werden vielschichtige kulturelle Praxiswelten, wie sie insbesondere für Krankenhäuser typisch sind, und gewachsene Strukturen als Kontext alltäglicher Prozesse kaum berücksichtigt. Das Gleiche gilt für nichtlineare Abläufe und Unerwartbarkeiten, mit denen Krankenhäuser mehr als andere Organisationen konfrontiert sind. Von diesen Verständnissen unterscheidet sich die evolutionäre Prozessoptimierung, indem sie insbesondere den letztgenannten Aspekten explizit Rechnung trägt.

\section{Charakterisierung einer «evolutionären Prozessoptimierung»}

Im Zentrum einer «evolutionären Prozessoptimierung» steht die Verbesserung der fachbereichsübergreifenden Zusammenarbeit und damit ein Aspekt, der oftmals als nicht veränderbar in den Hintergrund gedrängt wird. Ein solches Vorhaben will umsichtig angegangen werden und erfordert entsprechend Zeit. Evolutionäre Prozessoptimierungen sind langfristig angelegt und werden meist in Form strategischer Initiativen initiiert, die sich über einen Zeithorizont von mindestens zwei bis drei Jahren erstrecken.

Für eine abteilungsübergreifende Kooperation gerade im Kontext autonomer Professionsberei- che ist ein gemeinsames Verständnis über Art, Inhalt und Hintergründe der zu bewältigenden Verbesserungen und Herausforderungen unverzichtbar. Eine solche Vergemeinschaftung legt den Grundstein für eine erfolgreiche Prozessoptimierung. Hier werden möglichst viele der betroffenen Mitarbeitenden der unterschiedlichen Praxiswelten, das heisst Mitarbeitende der Pflege, Ärzteschaft und «Verwaltung», und Vertreter aller Hierarchieebenen in die Veränderungsanstrengungen integriert und an der Entwicklung einer Gesamtsicht sowie an der Ableitung und Umsetzung von Optimierungsmassnahmen beteiligt. Dadurch wächst die Akzeptanz für die bevorstehenden Veränderungen. Ein «Aussitzen» von Veränderungsprojekten, wie dies so häufig in Organisationen anzutreffen ist, ganz nach dem Motto «Auch das geht vorbei», wird unwahrscheinlicher.

Die Optimierungsmassnahmen wiederum sind bei weitem weniger radikal als im Falle eines BPR, gehen aber im Gegensatz zu Qualitätsund Zertifizierungsmassnahmen über eine blosse Dokumentation der bestehenden Abläufe hinaus. Ausgehend von «routinisierten Interaktionsmustern», also von Prozessen in ihrer konkreten, alltäglichen Ausprägung, wird explizit auf deren Verbesserung gezielt. Im Gegensatz zu Designmassnahmen wird dabei den gewachsenen Strukturen eines Krankenhauses explizit Rechnung getragen, sowohl auf sachanalytischer als auch auf emotional-sozialer Ebene. Gewachsene Strukturen existieren nicht umsonst und erfüllen vielfach eine wertvolle Funktion. Sie dürfen daher nicht pauschal durch neue ersetzt werden. So ist es beispielsweise nicht funktional, diagnostisch-therapeutische Prozesse beliebig zu standardisieren. Vielmehr muss sehr sorgfältig analysiert werden, welche Teile eines Prozesses als sinnvolle Einheiten zu bewahren sind und welchen Mustern diese Einheiten folgen. Aus diesen Einheiten können dann so genannte Prozessmodule entwickelt werden. Als standardisierte und optimierte Einheiten können diese idealerweise beliebig kombiniert werden und somit die komplexen und grundsätzlich unvorhersehbaren Abläufe abbilden und handhabbar machen.

Die Wirksamkeit einer «Evolutionären Prozessoptimierung» lässt sich insgesamt darauf zurückführen, dass es nicht zum radikalen Bruch mit organisationalen Routinen oder gewachsenen Denk- und Deutungsmustern kommt, sondern dass diese zunächst einmal sogar explizit in den Blick gebracht werden. Die geteilte Sicht lässt dann bei allen Beteiligten Organisationsbewusstheit wachsen, Darunter verstehen wir ein eingehendes Verständnis über die eigene Einbin- 
dung in die Gesamtwertschöpfung und über die wechselseitigen Abhängigkeiten, die über Bereichsgrenzen hinweg wirksam sind und massgeblich auf die eigene Arbeit einwirken. Während die Vergemeinschaftung essentiell ist für die Gestaltung und Umsetzung der Veränderungsanstrengungen, stellt Organisationsbewusstheit die Basis dar für eine später gut funktionierende $\mathrm{Zu}$ sammenarbeit über Bereichsgrenzen hinweg.

\section{Zusammenfassung}

Bisher wird das Thema «Prozessmanagement» im Krankenhausalltag relativ undifferenziert bearbeitet. Ein differenziertes Verständnis der alternativen Vorgehensweisen ist jedoch Voraussetzung für die Wahl einer angemessenen Alternative. Zudem sensibilisiert die differenzierte
Betrachtung dafür, dass mit jedem Vorgehen auch völlig unterschiedliche Herausforderungen für das Organisieren und Führen verbunden sind, je nachdem, ob es sich beispielsweise um eine Zertifizierung oder um eine Optimierung handelt.

Für den Bereich Krankenhaus bietet sich insbesondere im Hinblick auf die geforderten Effizienz- und Effektivitätssteigerungen der in diesem Artikel skizzierte Ansatz der «Evolutionären Prozessoptimierung» an. Wie genau die Umsetzung dieser Spielart des Prozessmanagements aussehen kann, welche Herausforderungen sich daraus für das Führen und Organisieren ergeben und wie diese Herausforderungen gehandhabt werden können, wird Gegenstand des Folgeartikels «Evolutionäre Prozessoptimierung» sein. 